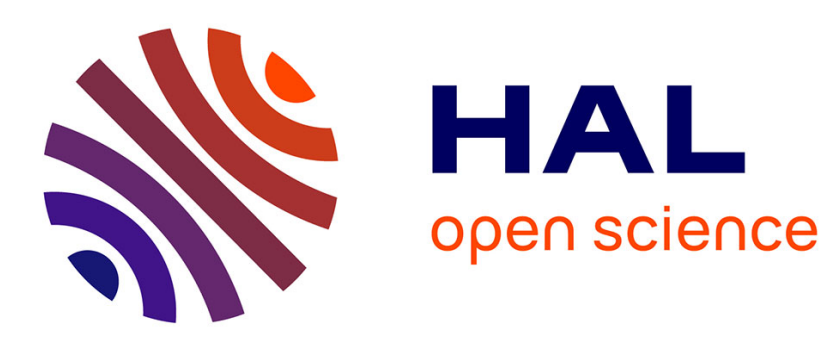

\title{
Interaction strength of weakly coupled pairs measured by a two laser spectroscopy technique
}

\author{
J.C. Vial, R. Buisson
}

\section{To cite this version:}

J.C. Vial, R. Buisson. Interaction strength of weakly coupled pairs measured by a two laser spectroscopy technique. Journal de Physique Lettres, 1982, 43 (10), pp.339-345. 10.1051/jphyslet:019820043010033900 . jpa-00232057

\section{HAL Id: jpa-00232057 https://hal.science/jpa-00232057}

Submitted on 1 Jan 1982

HAL is a multi-disciplinary open access archive for the deposit and dissemination of scientific research documents, whether they are published or not. The documents may come from teaching and research institutions in France or abroad, or from public or private research centers.
L'archive ouverte pluridisciplinaire HAL, est destinée au dépôt et à la diffusion de documents scientifiques de niveau recherche, publiés ou non, émanant des établissements d'enseignement et de recherche français ou étrangers, des laboratoires publics ou privés. 
Classification

Physics Abstracts

$78.50-78.55$

\title{
Interaction strength of weakly coupled pairs measured by a two laser spectroscopy technique
}

\author{
J. C. Vial and R. Buisson \\ Laboratoire de Spectrométrie Physique (*), \\ Université Scientifique et Médicale de Grenoble, B.P. 53 X, 38041 Grenoble Cedex, France
}

(Reçu le 17 février 1982, accepté le 22 mars 1982)

\begin{abstract}
Résumé. - Lorsque le couplage entre les deux ions d'une paire dépasse une certaine valeur, l'excitation des deux ions est impossible avec un seul laser fin. Cette double excitation est réalisée avec deux lasers de différente longueur d'onde. Un rétrécissement des raies inhomogènes est observé et apporte des informations sur le champ de contrainte dans le cristal. L'ordre de grandeur du couplage est déduit de la différence entre les longueurs d'onde et comparé à des taux de transferts assistés de phonons.
\end{abstract}

\begin{abstract}
When the coupling between two ions associated in pair exceeds a given value, the excitation of both ions of the pair is impossible when using a single narrow laser. This double excitation was obtained with two lasers of different wavelengths. A narrowing of the inhomogeneous broadened lines is observed which gives information on the strain field in the crystal. From the difference between the wavelengths, the order of magnitude of the coupling is deduced and compared with phonon assisted transfer rates.
\end{abstract}

The development of dye lasers has triggered new investigations of excitation transfer between ions in solids. $\mathrm{LaF}_{3}: \mathrm{Pr}^{3+}$ is an interesting system and has been used to study some fundamental aspects of transfer within an inhomogeneous line $[1,2]$ and also the delocalization of the excitation [3]. The results have stimulated theoretical analysis for explaining them $[4,5,6]$. Recently, a direct measurement of the transfer rates between two $\mathrm{Pr}^{3+}$ ions associated as a pair has been reported for some ten different classes of pairs [7]. That has been done by a selective excitation of a class of pairs using the fact that each class is associated, for each transition, with a satellite of the main line (due to isolated ions). These satellites are shown in figure 1 for the transition

$$
{ }^{3} \mathrm{H}_{4}(1) \rightarrow{ }^{1} \mathrm{D}_{2}(2) \text { of } \mathrm{Pr}^{3+} \text {. }
$$

Grossly, the shift of a satellite from the main line is an indication of the spatial distance between the two ions of the pair : the larger this shift, the nearer the ions. With weakly doped crystals $(0.02 \%$ to $0.2 \%)$, most of the pairs are " isolated » and thus their behaviour is not perturbed by a third ion.

(*) Laboratoire associé au CNRS. 


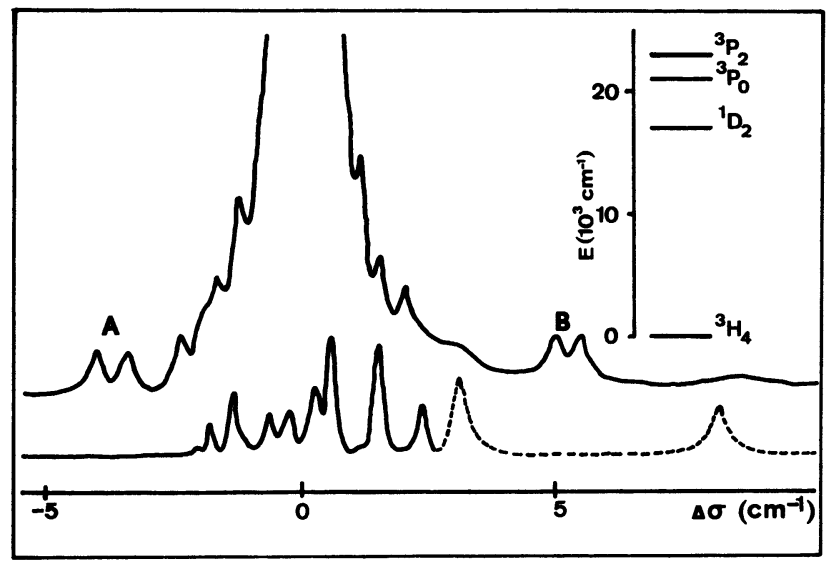

Fig. 1. - Top : Absorption spectrum around the transition ${ }^{3} \mathrm{H}_{4} \rightarrow{ }^{1} \mathrm{D}_{2}(2)$. Bottom : Excitation spectrum of the up-conversion fluorescence from ${ }^{3} \mathrm{P}_{0}$. On the right, energy levels of interest for $\mathrm{Pr}^{3+}: \mathrm{LaF}_{3}$.

The method used for the direct measurement of the transfer rate is based on the existence of an up-conversion transfer which occurs when both ions of a pair are excited. A blue fluorescence from ${ }^{3} \mathrm{P}_{0}$ is observed following a pumping into ${ }^{1} \mathrm{D}_{2}(2)$ with a yellow-orange pulsed laser. A time resolved excitation spectrum of this fluorescence obtained by scanning the laser wavelength is recalled in figure 1. The transfer rate for each class of pairs has been deduced from the dynamics of the fluorescence emitted by this class.

It can be seen in figure 1 that satellites $A$ and $B$ do not produce that up-conversion fluorescence. However, in the course of the studies of references [7, 8], that fluorescence was observed when using a laser with a broad spectral width. To try to clarify this point, experiments with two lasers of different wavelengths have been performed. Some preliminary results are given here.

The basic experimental arrangement is the same as that previously described [8], but here two dye lasers can excite a small volume of the $\mathrm{LaF}_{3}: \mathrm{Pr}^{3+}$ crystal. The $5 \mathrm{~ns}$ pulses of the two lasers have the same periodicity and can be simultaneous, or not. The two laser beams are well focused to increase the light intensities (the processes are bilinear in these intensities) and perfectly collinear, a critical setting not easy to obtain. The blue fluorescence resulting from this double excitation is monitored as a function of the wavelength of one laser, the wavelength of the other being fixed.

Regions A and B of figure 1 have first been studied as follow. The wavelength of laser $L_{1}$ is chosen in order to pump one of the satellites appearing in the absorption spectrum. Laser $\mathrm{L}_{2}$, whose pulses arrive on the crystal about $50 \mathrm{~ns}$ after the $\mathrm{L}_{1}$ pulses, is scanned in the spectral neighbourhood of the line excited by $\mathrm{L}_{1}$. The excitation spectra obtained by monitoring the up-conversion fluorescence are shown in figure 2. These spectra show that this fluorescence is created when the $L_{2}$ wavelength has nothing in common with an absorption line. If the pulses of laser $L_{2}$ arrive on the crystal before those of $\mathrm{L}_{1}$, the up-conversion fluorescence disappears. If the time delay between $L_{1}$ and $L_{2}$ pulses is increased, the intensity of that fluorescence decreases nearly exponentially with a time constant $\tau$ whose value for each of the four lines is given in table $\mathrm{I}$. In this table the rise time $\tau_{R}$ and the decay time $\tau_{D}$ of the observed fluorescences have also been given. From figure 2, it can also be pointed out that excitation lines are narrower than absorption lines and that if laser $L_{1}$ is slightly shifted from the centre of the absorption lines, the corresponding excitation line is shifted by the same value. This has been illustrated in figure 2 by showing that the differences $d_{1}, d_{2}, d_{3}$ between the energies of $\mathrm{L}_{1}$ and of the excitation line remain constant. (This is not perfectly true for lines associated with $B_{1}$ satellite.) When $L_{1}$ is just between $A_{1}$ and $A_{2}$, two 


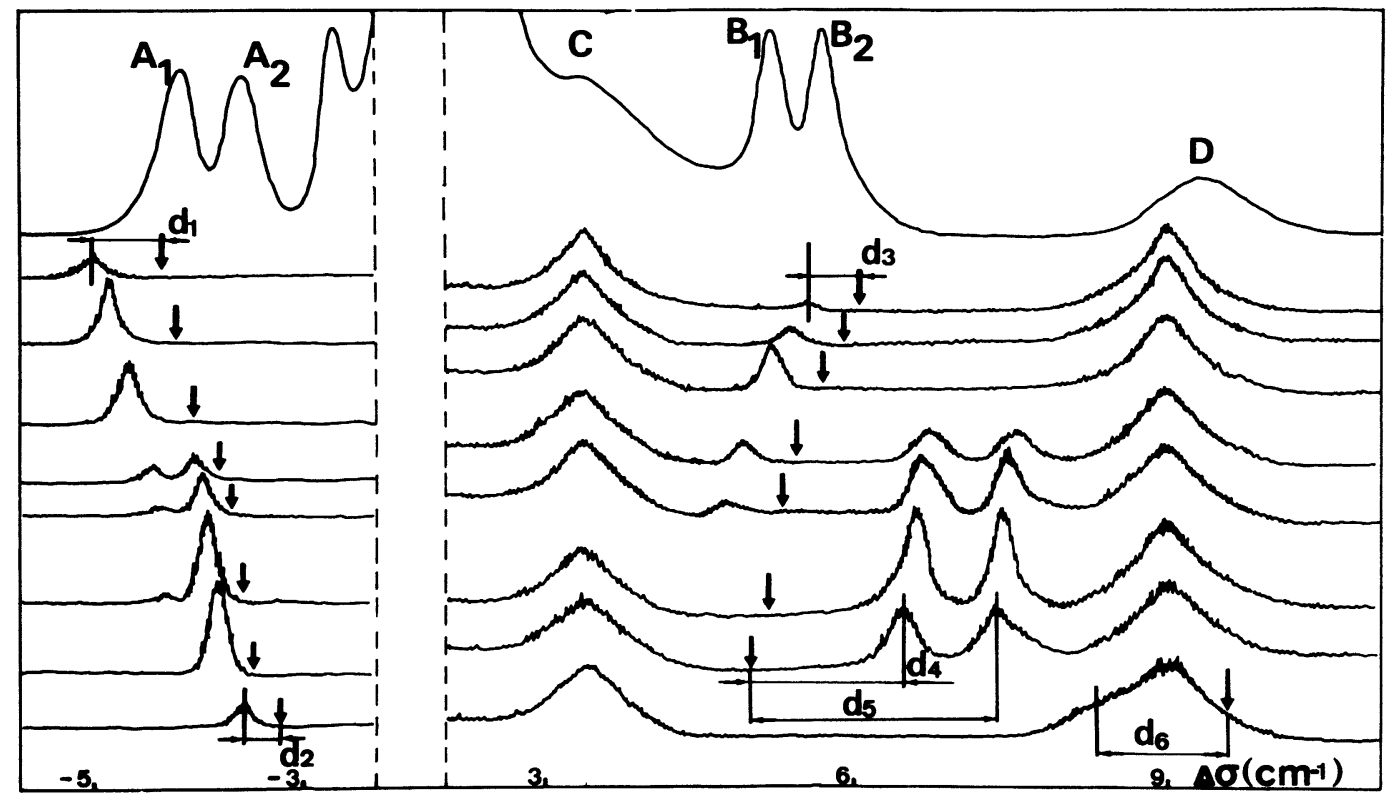

Fig. 2. - The upper lines recall the absorption spectrum of figure 1 with an extended energy scale. The other spectra are excitation spectra obtained by scanning laser $L_{2}$ when laser $L_{1}$ is at the position shown by the arrow. The energy differences $d_{1}, d_{2}, d_{3}$ between the energies of the lasers are constant when $\mathrm{L}_{1}$ position is changed, but $d_{4}$ and $d_{5}$ slightly change.

Table I. - Values (in $\mu \mathrm{s})$ for each line of the conversion fluorescence rise time $t_{\mathrm{R}}$ and decay time $t_{\mathrm{D}}$ and of the characteristic time $\tau$ of the variation of this fluorescence vs. the time separation between the pulses of the two lasers $\mathrm{L}_{1}$ and $\mathrm{L}_{2}$.

\begin{tabular}{ccrc} 
Line & $t_{\mathrm{R}}$ & $t_{\mathrm{D}}$ & $\tau$ \\
\hline- & - & -1 & - \\
$\mathrm{A}_{1}$ & $<1$ & 16 & 0.4 \\
$\mathrm{~A}_{2}$ & $<1$ & 30 & 0.4 \\
$\mathrm{~B}_{1}$ & 24 & 150 & 0.7 \\
$\mathrm{~B}_{2}$ & $<1$ & 18 & 0.2
\end{tabular}

excitation lines are observed, one associated with $A_{1}$, the other with $A_{2}$. Figure 2 shows also that two lines, shifted by $d_{4}$ and $d_{5}$ from the laser, are detected when satellite $\mathrm{B}_{1}$ is pumped by $\mathrm{L}_{1}$.

For $\mathrm{C}$ and $\mathrm{D}$ absorption lines, the situation is different since a single laser produces an upconversion fluorescence. Although these two lines seem to be very analogous (they are broader than the others, the excitation lines have long tails, the up-conversion rate is fast), the following observations have been possible only with $\mathrm{D}$ line. It is thought that this is due to the position of $\mathrm{C}$ line in the wing of the main line. The observations are :i) a shift between the absorption and the single laser excitation line; ii) the appearance of a "bump ", shifted by $d_{6}$ from the laser, on one side of the excitation line observed with laser $L_{2}$ when laser $L_{1}$ is pumping near the maximum of the absorption line (see the lower right spectrum in figure 2).

Before proposing an interpretation for these results, some data on $\mathrm{LaF}_{3}$ structure must be recalled. It seems now well accepted [9] that $\mathrm{LaF}_{3}$ belongs to the $\mathrm{P} \overline{3} \mathrm{Cl}\left(\mathrm{D}_{3 \mathrm{~d}}^{4}\right)$ space group, the $\mathrm{La}^{3+}$ sites, all identical, having a local symmetry $\mathrm{C}_{2}$ whose axis is perpendicular to the trigonal $C$ 
axis of the crystal [10]. Among the various « couples " of $\mathrm{La}^{3+}$ sites, some are such that the two sites are interchanged by a $C_{2}$ axis, others by inversion. These couples will be called symmetric. The other couples have none of these properties and will be called non symmetric. For instance, are symmetric the couples of sites in second, third and sixth neighbour positions with separations of respectively $4.26,4.33$ and $6.14 \AA$; are non symmetric the couples of sites in first, fourth and fifth neighbour positions with separations of respectively $4.1,4.43$ and $6.0 \AA$.

Considering first pairs of $\mathrm{Pr}^{3+}$ ions in symmetric sites, we can deduce from this symmetry property that the perturbations of the crystal field induced by each ion at the site of the other (and responsible of the shift of the satellites from the central line) are the same and thus that the energy levels of the two $\mathrm{Pr}^{3+}$ ions are identical. The Hamiltonian of a symmetric pair of class $(\alpha)$ made of two ions $a$ and $b$ can be written as

$$
\mathscr{H}_{\alpha}=\mathscr{H}_{\alpha}(\mathrm{a})+\mathscr{H}_{\alpha}(\mathrm{b})+\mathscr{H}_{\mathrm{c}}^{\alpha}(\mathrm{a}, \mathrm{b})
$$

where $\mathscr{H}_{\alpha}(\mathrm{i})$ is the crystal field Hamiltonian of ion (i), including the perturbation induced by the other, $\mathcal{H}_{\mathrm{c}}^{\alpha}(\mathrm{a}, \mathrm{b})$ the coupling Hamiltonian between the two ions.

Some of the energy levels of isolated $\mathrm{Pr}^{3+}$ ions have been shown in figure $3 a$. In fact, only three of them are of interest here : the ground state ${ }^{3} \mathrm{H}_{4}(1)$, the ${ }^{1} \mathrm{D}_{2}(2)$ state pumped by the laser due to

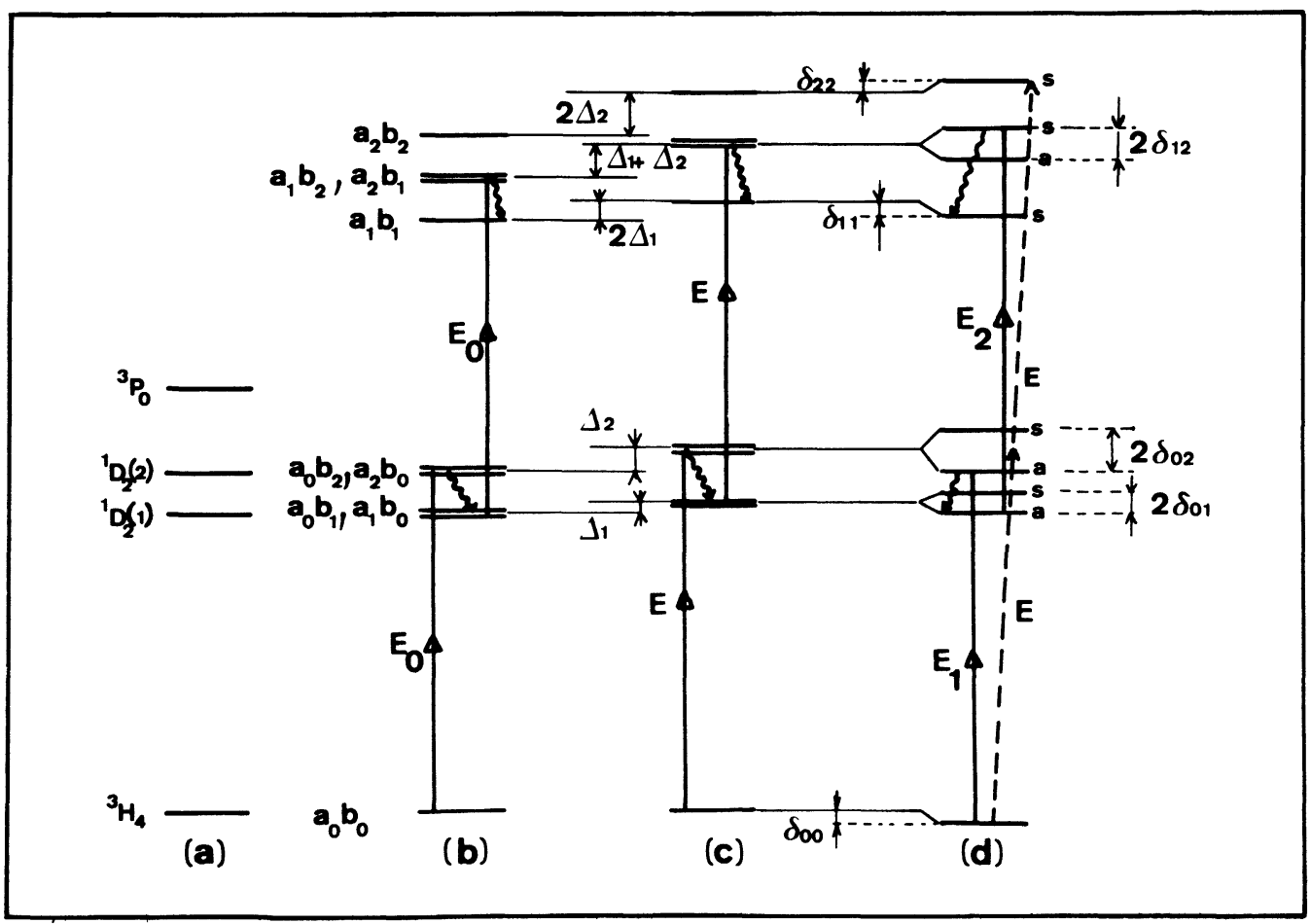

Fig. 3. - a) Energy levels of an isolated ion (the energy scale is not respected). b) Energy levels of a pair when neglecting the crystal field mutual perturbation and the coupling. Two photons $E_{0}$ can excite the pair twice. $c$ ) Energy levels of a pair when neglecting the coupling. $\Delta_{1}$ and $\Delta_{2}$ are the energy shifts induced by the crystal field mutual perturbation. Two photons $E$ can excite the pair twice. $d$ ) Energy levels of a pair of coupled ions. The $\delta_{\mathrm{ij}}$ are defined in the text. s and a mean symmetric and antisymmetric. Two photons $E$ can excite the pair twice by a double quantum transition. Two photons $E_{1}$ and $E_{2}$ can excite the pair twice by two successive one photon transitions. 
its strong oscillator strength and the ${ }^{1} \mathrm{D}_{2}(1)$ state to which ions in ${ }^{1} \mathrm{D}_{2}(2)$ relax with a characteristic time of about $10^{-10} \mathrm{~s}$ [11]. Let $\left|a_{0}\right\rangle,\left|a_{1}\right\rangle,\left|a_{2}\right\rangle$ be the eigenstates of $\mathcal{J}_{\alpha}(a)\left(\left|b_{0}\right\rangle,\left|b_{1}\right\rangle\right.$, $\left|b_{2}\right\rangle$ for $\left.H_{\alpha}(b)\right)$ corresponding respectively to ${ }^{3} H_{4}(1),{ }^{1} D_{2}(1)$ and ${ }^{1} D_{2}(2)$. Neglecting first the coupling term, the energy levels and the corresponding eigenstates of the pair have been shown in figure $3 b$ in the case when the mutual static perturbation is small and in figure $3 c$ when it is noticeable (as it is for the satellites visible on the spectra of figure 2): the $\Delta_{\mathrm{i}}$ are the energy shifts induced by this perturbation. The coupling term, $\mathcal{H}_{\mathrm{c}}^{\alpha}(\mathrm{a}, \mathrm{b})$, taken as a perturbation with respect to $\mathscr{H}_{\alpha}(\mathrm{a})+\mathscr{H}_{\alpha}(\mathrm{b})$ shows diagonal blocks when written in the $\left|\mathrm{a}_{l} \mathrm{~b}_{m}\right\rangle(l, m=0,1,2)$ basis because some unperturbed states, as for instance $\left|a_{1} b_{2}\right\rangle$ and $\left|a_{2} b_{1}\right\rangle$ are degenerated. The $2 \times 2$ block associated with this particular sub-space is

$$
\left|\begin{array}{cc}
E_{\alpha}+\left\langle a_{1} b_{2}\left|\mathcal{K}_{c}^{\alpha}\right| a_{1} b_{2}\right\rangle & \left\langle a_{1} b_{2}\left|H_{c}^{\alpha}\right| a_{2} b_{1}\right\rangle \\
\left\langle a_{2} b_{1}\left|H_{c}^{\alpha}\right| a_{1} b_{2}\right\rangle & E_{\alpha}+\left\langle a_{2} b_{1}\left|H_{c}^{\alpha}\right| a_{2} b_{1}\right\rangle
\end{array}\right|
$$

whose eigenvalues can be written as

$$
E_{\alpha} \pm \delta_{12}^{\alpha}
$$

and the eigenstates as

$$
\frac{1}{\sqrt{2}}\left[\left|a_{1} b_{2}\right\rangle \pm\left|a_{2} b_{1}\right\rangle\right]
$$

written shortly $|1,2\rangle_{\mathrm{S}}$ and $|1,2\rangle_{\mathrm{A}}$ respectively for the + and - signs.

The nine eigenstates of a pair are shown in figure $3 d$ where

$$
\delta_{l l}^{\alpha}=\left\langle a_{l} b_{l}\left|H_{c}^{\alpha}\right| a_{l} b_{l}\right\rangle \quad(l=0,1,2)
$$

and where the relative positions of the symmetric and antisymmetric eigenstates have been arbitrarily chosen.

This energy diagram is much simpler than comparative diagrams obtained for instance with pairs of $\mathrm{Cr}^{3+}$ in ruby [12] or pairs of $\mathrm{Nd}^{3+}$ in $\mathrm{LaCl}_{3}$ [13]. This results from the fact that the energy levels of these ions, in the absence of coupling, are degenerated and that the coupling between the two ions of a pair lifts the degeneracy, leading to a complicated energy diagram. With $\mathrm{Pr}^{3+}: \mathrm{LaF}_{3}$, since the energy levels of the isolated ions are singlets, the coupling leads to the well known symmetric (S) and antisymmetric (A) above eigenstates in which an excitation is delocalized on the two ions of the pair. This situation is quite similar with that encountered in molecular crystals, the pairs described here being then called dimers [14].

The transition $0 \rightarrow 2\left({ }^{3} \mathrm{H}_{4}(1) \rightarrow{ }^{1} \mathrm{D}_{2}(2)\right)$ induced by the laser is $\Pi$ polarized, i.e. the $\mathbf{E}$ vector of the light is parallel to the $c$ axis of the crystal. Symmetric pairs made of ions in second neighbour position have a twofold axis perpendicular to the crystal $c$ axis, those made of ions in third and sixth neighbour position (see above) have an inversion centre. Thus, for both cases, only symmetric $\leftrightarrow$ antisymmetric transitions can be excited by a $\Pi$ polarized light.

It is clear, from figures $3 c$ and $3 d$, that the double excitation of a pair, needed for an up-conversion transfer, is possible with a single laser only for weakly coupled pairs. That explains why most of the blue excitation lines observed with a single laser are near the centre of the main line (see Fig. 1). The double excitation with a single laser might also be obtained for more coupled pairs by a double quantum transition (see Fig. $3 d$ ). Except perhaps for lines $\mathrm{C}$ and $\mathrm{D}$ discussed below no evidence for this process has been found. 
The results obtained with two lasers for the strongly coupled pairs associated with lines $A_{1}, A_{2}$, $B_{2}$ are well explained from figure $3 d$ : laser $L_{1}$ is absorbed at a wavelength corresponding, for one satellite, at the transition from the symmetric ground state to the $|0,2\rangle_{\mathrm{A}}$ state ; after the fast relaxation to $|0,1\rangle_{A}$ (this point will be discussed elsewhere) the pair is excited by $L_{2}$ to $|1,2\rangle_{S}$; another fast relaxation puts it into $|1,1\rangle_{\mathrm{S}}$ from which the blue fluorescence occurs. The difference between the energy of the two photons is calculated from figure $3 d$ as

$$
E_{2}-E_{1}=\delta_{12}+\delta_{01}+\delta_{02}-\delta_{00}
$$

This difference gives thus the order of magnitude of the coupling Hamiltonian $\mathscr{H}_{\mathrm{c}}$. The determination of all the $\delta_{\mathrm{ij}}$ should need experiments with other polarization for the laser beams and also experiments by pumping into the lowest level of the ${ }^{1} \mathrm{D}_{2}$ multiplet. Due to the weakness of the oscillation strength for these transitions, lasers having larger power are necessary.

The analysis of the spectra shown in figure 2 leads to two interesting points. Firstly, it shows that the excitation lines are narrower than the absorption lines, the narrowing being probably limited in the experiments by both the laser width and the homogeneous width of the transition. This narrowing must be distinguished from that observed by the fluorescence line narrowing [15]. It has something in common with the "absorption line narrowing " observed in gases $[16,17]$ : the first laser selects a class of strains instead of a class of velocity in gases, and the second laser is absorbed only by the pairs already excited as in gases it is absorbed only by the excited atoms. Secondly, the actual observation of this narrowing gives the proof that the two ions of the pair are submitted to nearly the same strain. This is compatible with the fact that the broadening results essentially from strains induced by the $\operatorname{Pr}^{3+}$ ions themselves. Since the crystals used are weakly doped, the pairs are nearly isolated and thus far from the sources of the strains.

Finally, within this model, the time $\tau$ given in table I appears as the lifetime of the $|0,1\rangle_{A}$ state. It is short because, for a strongly coupled pair, a very fast de-excitation process occurs when only one ion of the pair is excited. Known as " fluorescence quenching " or " cross relaxation ", this process consists of a partial transfer of the excitation of this ion to its unexcited neighbour; the measure of $\tau$ gives its rate. The existence of this process is usually deduced from the non exponential decay of the fluorescence, but, as all the classes of pairs contribute, only " average effects » can be sorted out from this decay. The experiments reported here give the first direct measurement for the more strongly coupled pairs.

Whereas the results obtained with $A_{1}, A_{2}$ and $B_{2}$ lines are well explained within the above model, the results concerning $B_{1}$ and $D$ lines need further investigations. $B_{1}$ line is particularly puzzling because its strong shift from the central line suggests the two ions of the pair are near each other although the slow dynamics (see table I) suggests the opposite. It gives also particular results with the two laser experiments since two excitation lines by $\mathrm{L}_{2}$ are observed for one transition excited by $L_{1}$ (see Fig. 2). No « realistic » explanation has been found yet.

Results for the $\mathrm{D}$ line can be explained within the above model along the following arguments. This line, strongly shifted, can be associated with pairs made of ions in first neighbour position. These pairs being non symmetric, two terms must be added to the Hamiltonian (1), namely $\mathcal{H}_{\alpha \mathrm{s}}(\mathrm{a})+\mathcal{H}_{\alpha \mathrm{s}}(\mathrm{b})$, to describe the crystal field perturbation induced by each ion on the other. Then, the degeneracy of levels of figure $3 c$ is lifted and, for instance, the shift $\Delta_{2}$ takes two values $\Delta_{2}$ (a) and $\Delta_{2}($ b). However, since only one absorption line is observed (see Fig. 1) the difference $\Delta_{2}(\mathrm{a})-\Delta_{2}(\mathrm{~b})$ is smaller than the linewidth. Then, the above model can again be applied if the width is supposed to be homogeneous : the pair is then quasi-symmetric and the " bump " observed on the edge of $\mathrm{D}$ line in the two lasers experiments results from two successive absorptions of photons of $L_{1}$ and $L_{2}$. The shift between the absorption line and the bump(slightly smaller than $d_{6}$ in figure 3 ) gives again an order of magnitude of the coupling between the two ions. The upconversion line observed when using a single laser results from the overlap between the absorption 
curve and the "bump " which corresponds to the absorption of the second photon. Further experiments and analysis are necessary to clarify some points, particularly to understand why the homogeneous width of this $\mathrm{D}$ line (and perhaps also that of $\mathrm{C}$ line) is much larger than that of the other lines.

Although some points have yet to be clarified, as the nature of the coupling between ions of strongly coupled pairs (which is probably not dipole-dipole as shown in reference [7]) or the attribution of the absorption lines to the various classes of pairs, we want to point out that the preliminary results reported here show the interest of the technique used and bring physical information. The technique is a two-photon absorption technique, well known in gas spectroscopy but applied to solids for the first time. It has the advantage to narrow broadened lines inhomogeneously and has given here information on the field strain. It has also made possible for the first time to measure both static and dynamic consequences of the coupling between two ions associated in pairs. Leaving a more quantitative analysis for a forthcoming paper, one can recall the order of magnitude : energy shifts of the order of $0.5 \mathrm{~cm}^{-1}\left(1.5 \times 10^{10} \mathrm{~Hz}\right)$ and phonon assisted transfer rates of $10^{7} \mathrm{~s}^{-1}$. From the former a resonant transfer rate of $10^{10} \mathrm{~s}^{-1}$ can be deduced. The ratio between these two rates shows the reduction introduced by the participationof a phonon for the transfer.

Acknowledgments. - It is a pleasure for the authors to thank F. Madéore for his technical assistance, particulary in the achievement of the performances of the lasers, and R. Romestain for very stimulating discussions.

\section{References}

[1] Flash, R., Hamilton, D. S., Selzer, P. M., Yen, W. M., Phys. Rev. Lett. 35 (1975) 1034.

[2] Flash, R., Hamilton, D. S., Selzer, P. M., Yen, W. M., Phys. Rev. B 15 (1977) 1248.

[3] Hegarty, J., Huber, D. L., Yen, W. M., Phys. Rev. B 23 (1981) 6271.

[4] Holstein, T., Lyo, S. K., Orbach, R., Phys. Rev. B 15 (1977) 4693.

[5] Holstein, T., Lyo, S. K., Orbach, R., Phys. Rev. B 16 (1977) 934.

[6] Huber, D. L., Hamilton, D. S., Barnett, B., Phys. Rev. B 16 (1977) 4642.

[7] Buisson, R., Vial, J. C., J. Physique Lett. 42 (1981) L-115.

[8] Vial, J. C., Buisson, R., MadÉore, F., Poirier, M., J. Physique 40 (1979) 913.

[9] Cheetham, A. K., Fender, B. E. F., Fuess, H., Wright, A. F., Acta Crystallogr. B 32 (1976) 94.

[10] Von Mansmann, M., Z. Kristall. 122 (1965) 375.

[11] Erickson, L. E., Phys. Rev. B 11 (1975) 77.

[12] Kisliuk, P., Chang, N. C., Scott, P. L., Pryce, M. H. L., Phys. Rev. 184 (1969) 367.

[13] Prinz, G. A., Cohen, E., Phys. Rev. 165 (1968) 335.

[14] FöRSTER, T., in Modern Quantum Chemistry, part. III ; ed. by O. Sinanoglu (Academic Press, N.Y.), 1965, Ch. B. 1.

[15] Szabo, A., Phys. Rev. Lett. 27 (1971) 323.

[16] Delsart, C., Keller, J. C., Opt. Commun. 15 (1975) 91.

[17] Delsart, C., Keller, J. C., Opt. Commun. 16 (1976) 388. 